\title{
Modelling and Characterization of Cell Collapse in Aluminium Foams During Dynamic Loading
}

M. A. Kader ${ }^{1}$, M. A. Islam ${ }^{1}$, P. J. Hazell ${ }^{1, *}, J^{*}$ P. Escobedo ${ }^{1}$, M. Saadatfar ${ }^{2}$, A. D. Brown ${ }^{1}$ and G. J. Appleby-Thomas ${ }^{3}$

${ }^{1}$ School of Engineering and Information Technology, The University of New South Wales, Canberra, ACT 2600, Australia

${ }^{2}$ Department of Applied Mathematics, Australian National University, Canberra, ACT-0200, Australia

${ }^{3}$ Cranfield Defence and Security, Cranfield University, Shrivenham, Swindon SN6 8LA, UK *Corresponding Author, E-mail: P.Hazell@adfa.edu.au

\section{Highlights}

Plate impact experiment have been carried out on closed-cell aluminium foams

A micro-computed tomography based geometry was developed for modelling studies

Wave propagation mechanism through the closed-cell $\mathrm{Al}$ foams has been investigated

$>$ Cell collapse mechanism has been explored during shock loading

\begin{abstract}
Plate-impact experiments have been conducted to investigate the elastic-plastic behaviour of shock wave propagation and pore collapse mechanism of closed-cell aluminium foams. FE modelling using a meso-scale approach has been carried out with the FE software
\end{abstract}


ABAQUS/Explicit. A micro-computed tomography-based foam geometry has been developed to reproduce the behaviour during shock loading. Microstructural changes with time have been investigated to explore the effects of wave propagation with special attention given to the pore-collapse mechanism. The effect of velocity variations on deformation has been elucidated with three different impact conditions using the plate-impact method. Free surface velocity $\left(u_{f s}\right)$ was measured to understand the evolution of the compaction. The freesurface velocity increased gradually at lower flyer-plate velocities, whereas an abrupt rises of free-surface velocity was found at $845 \mathrm{~m} / \mathrm{s}$ with a copper flyer-plate which reflects the appearance of shock. A good correlation was found between experimental results and FE predictions.

\section{Keywords}

Shock propagation; Closed-cell aluminium foam; Plastic collapse; Micro-computed tomography; Meso-scale modelling

\section{Introduction}

For more than two decades, closed-cell aluminium foams have been used in protective applications to attenuate impact and shock effects [1, 2]. High impact energy absorption capacity and good shock wave attenuation due to the complex deformation of the material [3, 4] makes these materials attractive compared to traditional metals. Their main advantage during impact loading is that they transmit relatively low amounts of load due to a unique non-linear plastic deformation process [5]. The applications of these materials are increasing day by day in different sectors such as military equipment $[6,7]$, automotive parts [8], structural elements and aerospace [4, 9-11]. However, little attention has been given to the 
shock-wave attenuation and the energy absorption mechanism of metal foams particularly where the foam has been struck by a projectile $[12,13]$. Moreover, a number of previous studies confirm that the plastic deformation of porous materials owing to high velocity impact loading significantly depends on the nature of the shock loading $[2,9,13,14]$. To understand the nature of shock compaction and pore collapse in these materials it is essential to probe the dynamic response using finite-element techniques. This is due to the enormous complexities of observing small structures deforming in short-time scales [15].

A few earlier $[5,16]$ and recent studies $[2,9,13]$ have elucidated the deformation behaviour of different cellular materials under impact loading. However, the majority of these works are based on low velocity impact where shock compaction of the material does not occur. Tan et al. [14] studied the shock response of an open-cell aluminium foam experimentally and analytically. In that work they measured the plastic collapse stress in different loading regimes. They concluded that the plastic collapse of the metal foam was due to wave propagation. However there is still a scarcity of information on how the wave navigates through the porous structure and the nature of pore collapse. Recently, Petel et al. [2] investigated the shock response of an open-cell aluminium foam through plate-impact experiments. They observed a precursor wave at the beginning of loading in their plateimpact experiment. Similarly, another research group [5] predicted a precursor wave using analytical approaches. They concluded that in the elastic region, a precursor wave appears in both the subsonic and sonic regimes whereas it disappears in the supersonic regime.

Several groups of researchers have developed various techniques to investigate the shock propagation through metal foams and cellular materials using numerical modelling approaches [17, 18], analytical methods [1, 19, 20] and 1-D shock-wave modelling [21, 22]. However, most of previous researchers overlook the complex geometrical structure and localized deformation of the cell walls that will significantly affect the predicted results. It 
can be noted that, previously significant studies have been carried out to investigate the impact and shock response of solid metals [23-28] and ceramics [29]. In contrast, there is a paucity of similar data for metal foams. In spite of having a significant structural influence on dynamic behaviour, most of the existing research $[17,18,30]$ is devoted to continuum or multi-cell approaches for developing the foam geometry. However this continuum-based research was not able to visualise the actual wave propagation through the convoluted network of cell walls. A few research groups [30,31] have developed the foam geometry with homogeneous distribution of regular shape voids although the actual foam consists of completely non homogeneous distribution of irregular shape voids. To the authors' best knowledge only Bourne et al. [32] has investigated the shock response of aluminium foams through plate-impact experiment using micro-tomography based real geometry. However, they investigated the open-cell foams and their study cannot show the wave propagation and details pore collapse mechanism during shock loading.

In this study, a suite of plate impact experiments were conducted on closed-cell aluminium foams with three different impact velocities. Moreover, numerical simulations employing a meso-scale approach have been carried out to critically explore elastic-plastic wave propagation and local pore collapse mechanisms. X-ray computed micro-tomography-based foam geometry has been developed to reveal important insights during loading. A MieGruneisen equation-of-state (EOS) with linear $\mathrm{U}_{\mathrm{s}}-\mathrm{u}_{\mathrm{p}}$ and a Johnson-Cook plasticity material model with dynamic failure are used to describe the material behaviour in the meso-scale modelling. Extensive dynamic simulations were carried out to investigate the effects of intense impact, and the associated free surface velocity $\left(U_{f s}\right)$.

\section{Experimental Methods}

\subsection{Material used}


In this study we used aluminium foams (closed-cell) from CYMAT Aluminium Corporation with a nominal density of $0.5 \mathrm{~g} / \mathrm{cc}$. Electro Discharge Machining (EDM) was used to cut the materials to make a number of 10 -mm thick samples whilst avoiding cell-wall distortion. Furthermore, the experimental samples were picked from a number of pieces considering the homogeneity of pore distribution and sizes. The authors previously measured the physical properties of this material and the data is presented in Table 1 [33]. The chemical compositions of the selected closed-cell aluminium foam were also measured by energy dispersive spectroscopy (EDS) analysis. It was reported that the foam material contained trace amounts of $\mathrm{Si}, \mathrm{O}, \mathrm{Fe}$ and Ti elements with aluminium [33].

Table 1. Physical properties of closed-cell aluminium foam [33]

\begin{tabular}{|c|c|}
\hline Properties & Measured values \\
\hline Density (g/cc) & $0.49 \pm 0.02$ \\
\hline Relative density (\%) & $17.50 \pm 0.51$ \\
\hline Porosity (\%) & $82.00 \pm 0.53$ \\
\hline Mean Cell-size (mm) & $1.75 \pm 1.13$ \\
\hline Wall thickness (mm) & $0.17 \pm 0.08$ \\
\hline
\end{tabular}

A detailed topological and morphological analysis for this foam has also been carried out by micro-computed tomography [34]. The sample material photographs in Figure 1 show that the distribution of pores through the foam thickness is not homogeneous. The uniaxial stressstrain response of the aluminium foam shown in Figure 2 was reported in authors' previous work [33]. 


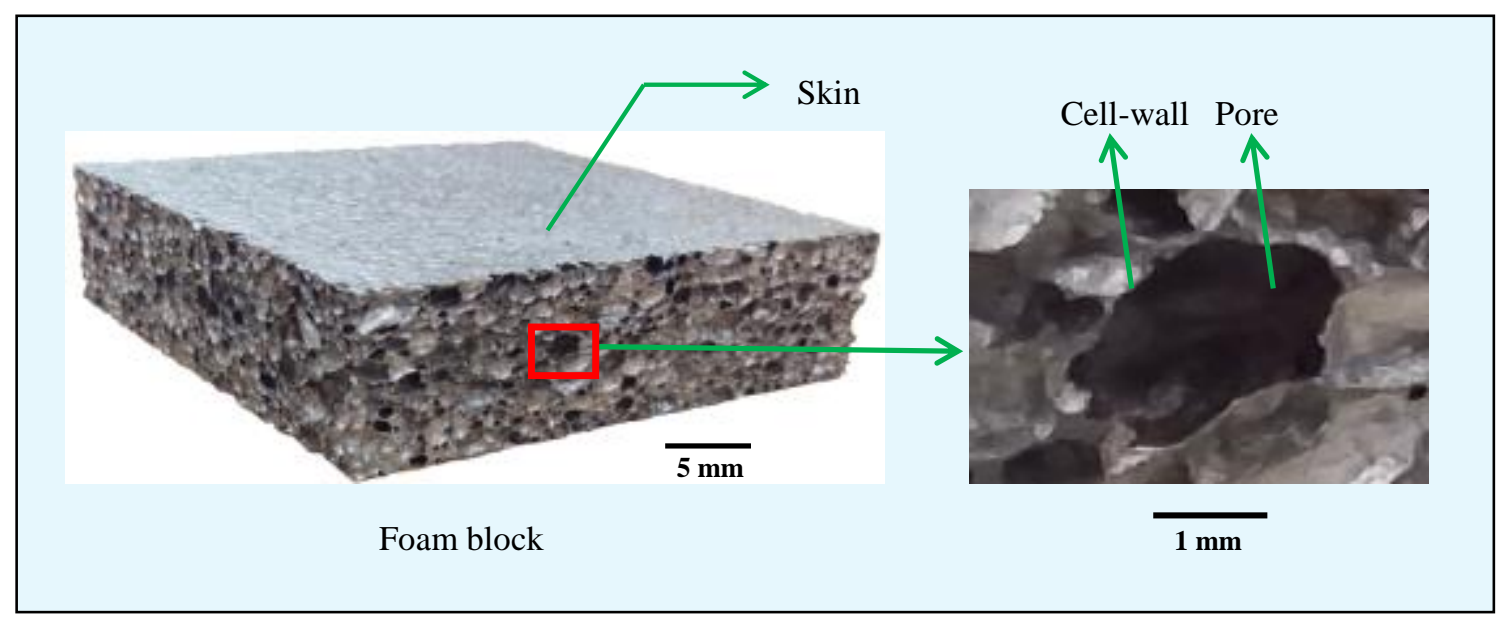

Figure 1- Photograph of foam sample with cell-wall and pore.

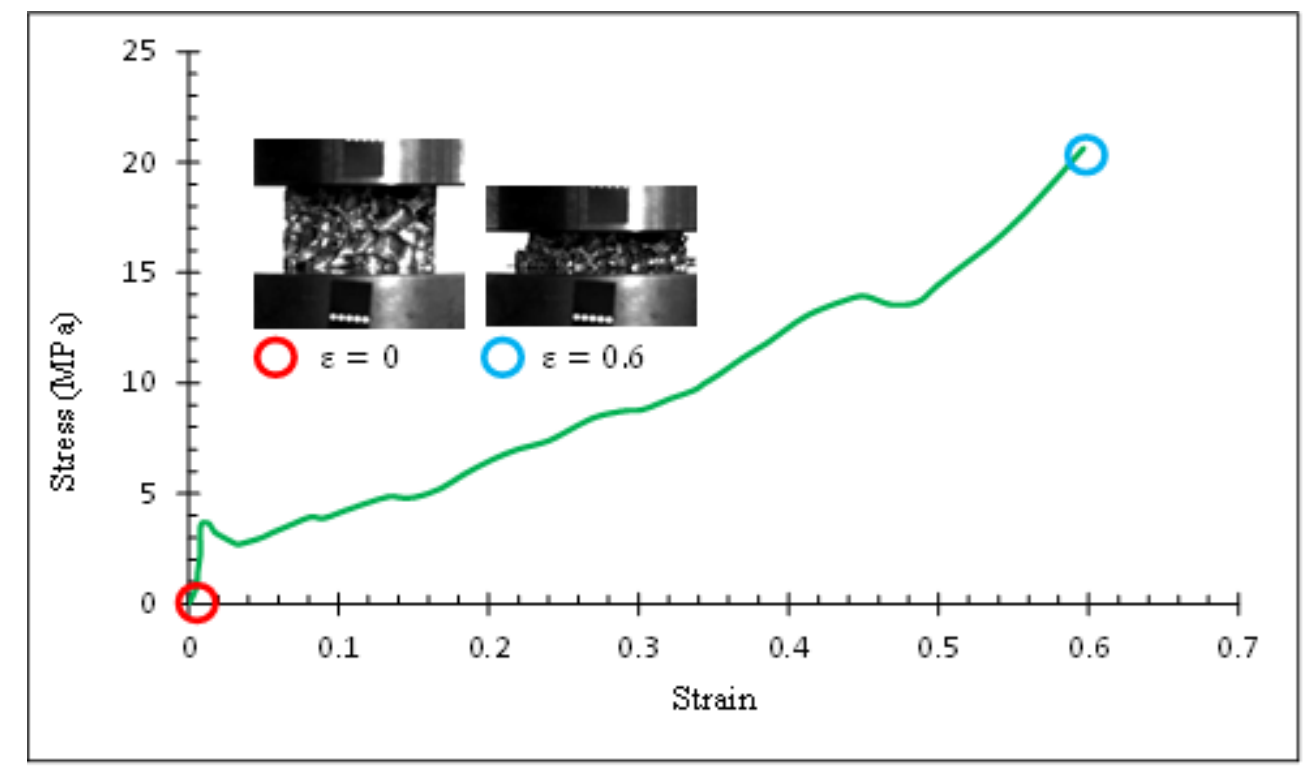

Figure 2- Quasi-static stress-strain response of closed-cell aluminium foam of density $0.5 \mathrm{~g} / \mathrm{cc}$ [34].

\subsection{Experimental Methods}

Plate-impact experiments were performed at Cranfield University with a $5 \mathrm{~m}$ single-stage gas gun. A number of experiments were carried out with three different impact velocities. 
Aluminium and copper flyer plates of $10 \mathrm{~mm}$ thickness were used to impact the target samples. A copper flyer-plate was used for higher impact velocity $(845 \mathrm{~m} / \mathrm{s})$ whereas aluminium flyers were used for comparatively lower impact velocities (480 m/s and $219 \mathrm{~m} / \mathrm{s}$ ). In each case the flyer-plates were lapped flat and parallel to a tolerance of $5 \mu \mathrm{m}$ before being mounted onto an acetal sabot (see Figure 3).

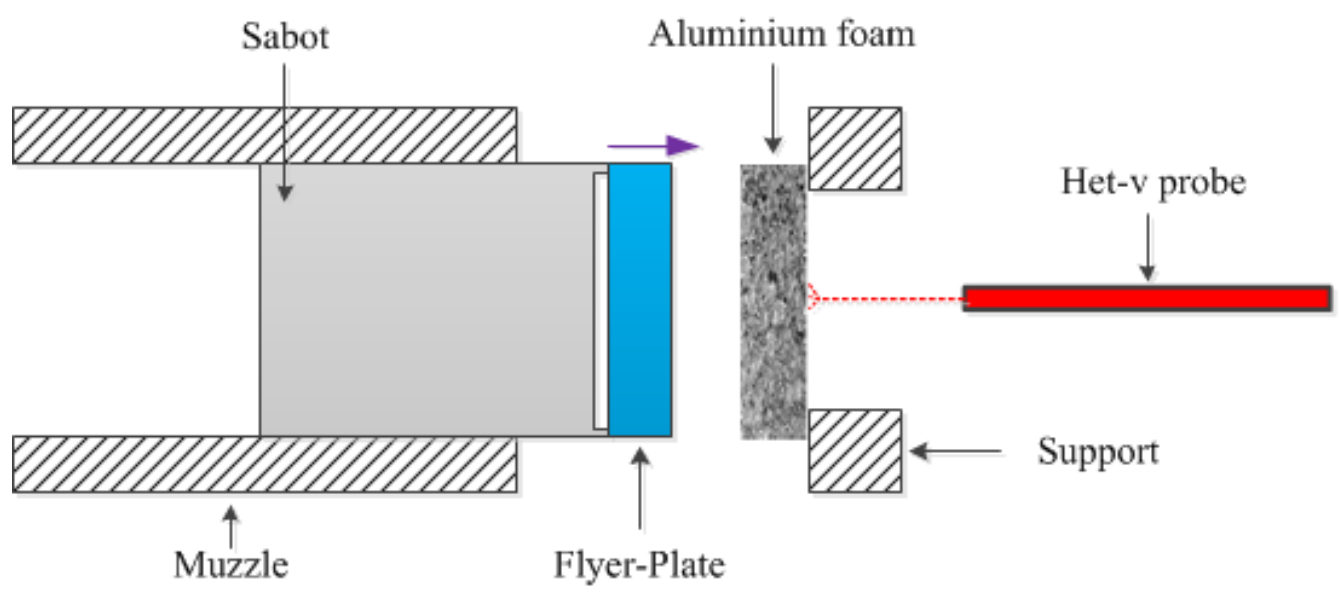

Figure 3-Schematic of flyer-plate impact experiment.

A Heterodyne velocimeter (Het-v) was used to measure the free surface velocity. The Het-v was placed at 30 millimetres apart from rear face of target aligning with its centre. A schematic of plate-impact experiment is shown in Figure 3.

The diagnostics system employed a $12 \mathrm{~mW}$ laser source (DFB-1550-BF-20-2.5-FA) operating at $1545 \pm 15 \mathrm{~nm}$. An ITC-510 Laser Diode combi-controller was used to control both the temperature and the supply current to the laser diode, ensuring a stable output wavelength. The interfered light was collected by a PDA8GS amplified photodetector, having $8 \mathrm{GHz}$ bandwidth. A disposable experimental probe was employed consisting of a 
collimating lens connected to a $5 \mathrm{~m}$ length of $9 / 125 \mu \mathrm{m}$ single-mode fibre. In these studies, the raw oscilloscope data from the interferometer were processed with MATLAB using a Gabor transform to produce a time-frequency spectrogram. The spectrogram images were then processed using a standard image manipulation application, Image $\mathrm{J}$, to manually select points on the plot and digitise those into $(\mathrm{X}, \mathrm{Y})$ pairs for subsequent data reduction using Microsoft Excel.

\section{Numerical Methods}

\subsection{Geometry development}

The digital foam geometry was developed with X-ray computed micro-tomography. It is a non-destructive technique that allows a complete 3D internal structure of an opaque object to be determined by reconstructing the X-ray absorption coefficients of materials. A flat panel detector and micro-focus X-ray source of $100 \mathrm{kV}$ were used for imaging. The foam specimen was placed on a motor controlled rotating stage and radioscope projections were taken after each degree of rotation. Subsequently, the grey projections were transferred to binary images and processed to reconstruct full 3D digital foam geometry. Tetrahedral elements were used to mesh the geometry due to complex geometrical structure. The flow chart of the complete process is shown in Figure 4. Then the meshed geometry was converted to ABAQUS friendly format and imported to ABAQUS/Explicit for simulations. The developed foam geometry with its sectional view is shown in Figure 5. 


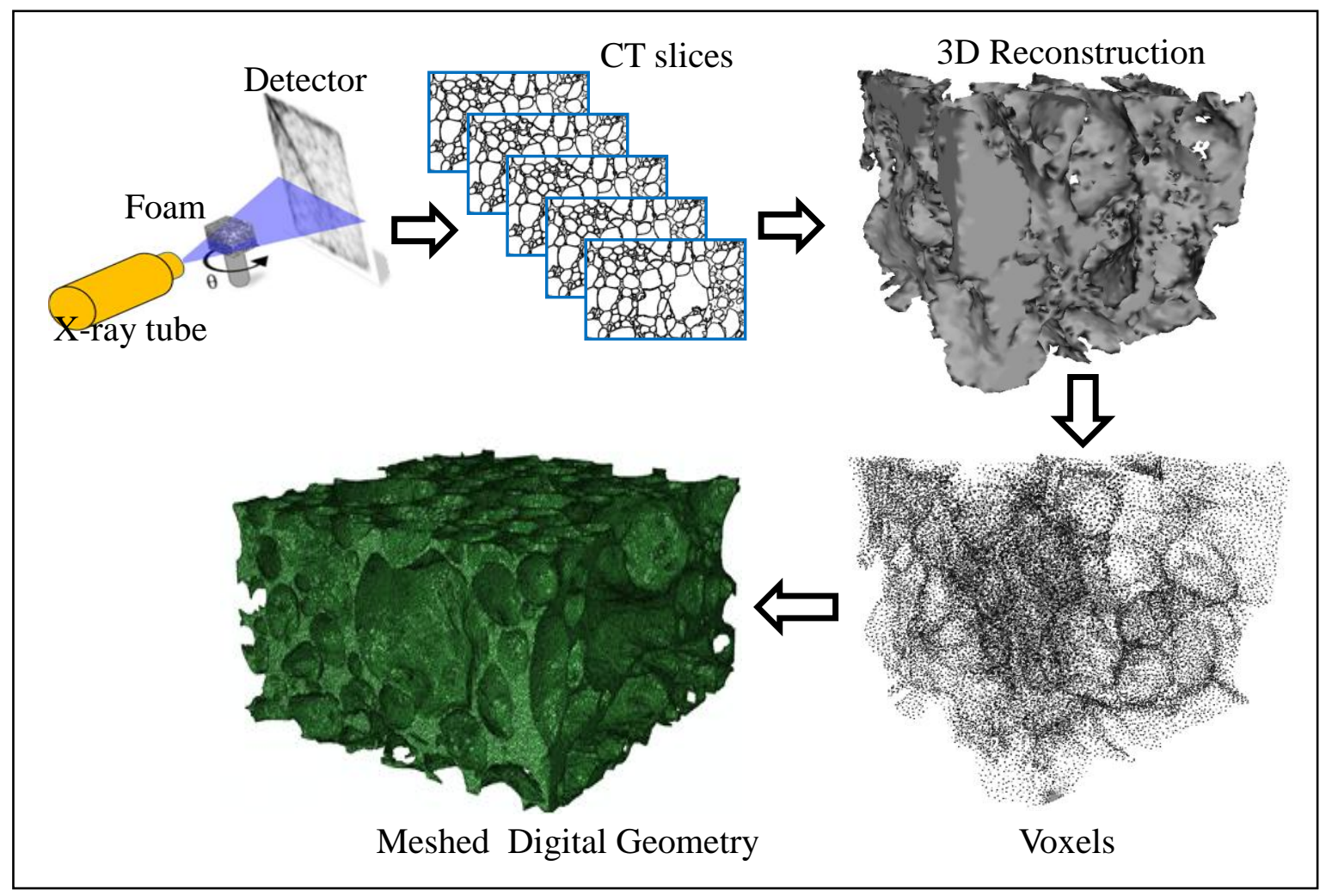

Figure 4- Flow diagram of micro-CT based foam geometry development.

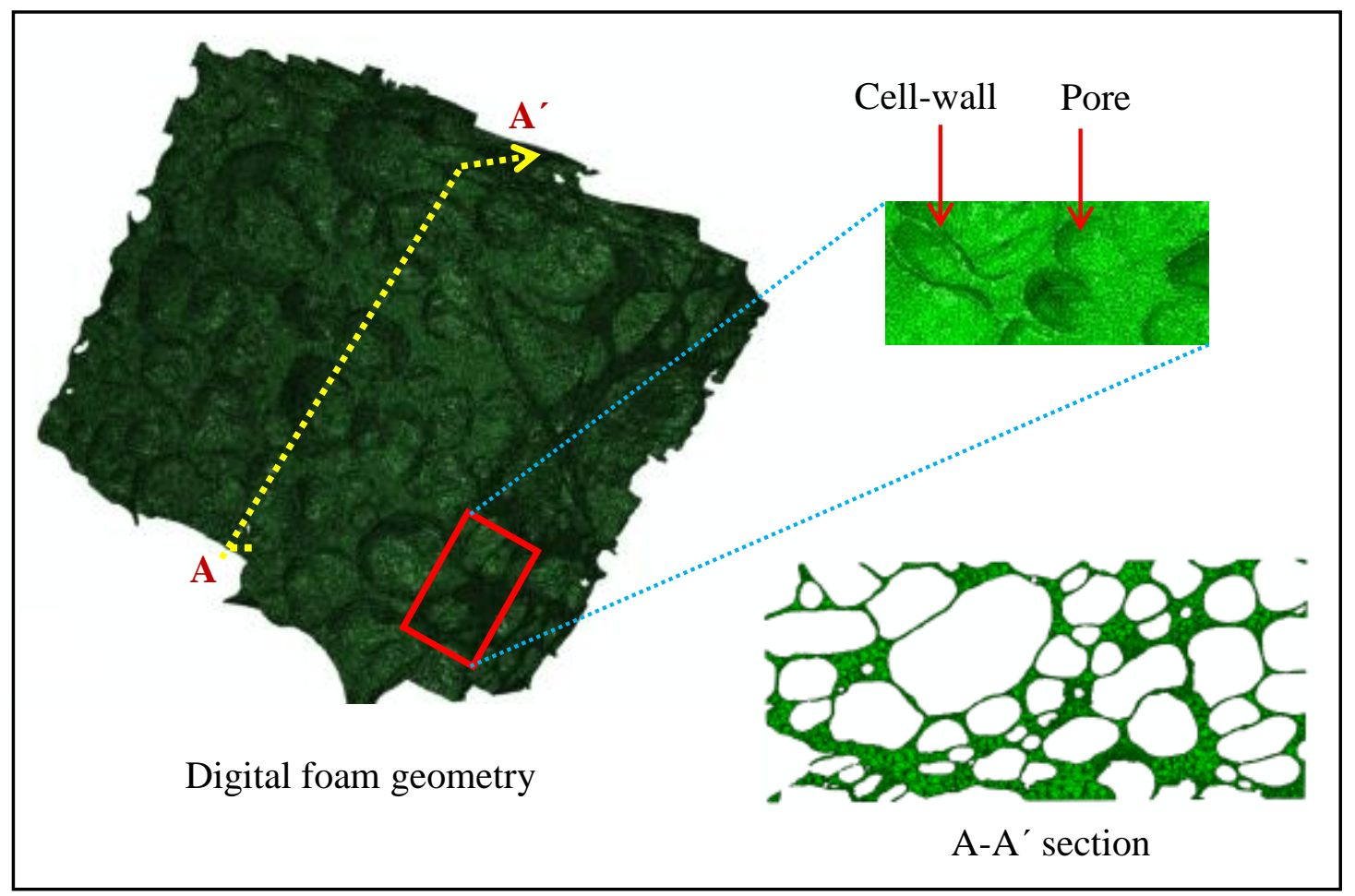

Figure 5- Developed 3D digital foam geometry and sectional view. 


\subsection{Finite Element Modelling}

The FE software ABAQUS/Explicit was used to perform the simulation. The modelling has been carried out to probe the nature of wave propagation and the pore-collapse mechanisms. These are currently difficult to do with experimental techniques. Micro-computed tomography based dynamic modelling were performed to explicitly describe the shock effects. The Mie-Gruneisen Equation of State (EOS) with a linear shock velocity to particle velocity relationship, $\mathrm{U}_{\mathrm{s}}-\mathrm{u}_{\mathrm{p}}$ was used to describe the material properties of the aluminium that makes up the foam. This is given by

$$
\mathrm{P}=\frac{\rho_{0} \mathrm{c}_{0}^{2} \eta}{(1-\mathrm{s} \eta)^{2}}\left(1-\frac{\Gamma_{0} \eta}{2}\right)+\Gamma_{0} \rho_{0} \mathrm{E}_{\mathrm{m}}
$$

where the reference density, $\rho_{0}=2700 \mathrm{~kg} \mathrm{~m}^{-3}$, the bulk sound speed, $\mathrm{c}_{0}=5.24 \mathrm{~mm}^{-1}$, Hugoniot slope, $s=1.40$ and Gruneisen coefficient, $\Gamma_{0}=1.9$ [35]. An elastic, Johnson-Cook plasticity (Equation 2) material model with Johnson-Cook dynamic failure criteria for the structural material (Al) was applied to simulate the plastic response [36].

$$
\sigma^{0}=\left[\mathrm{A}+\mathrm{B}\left(\bar{\varepsilon}^{\mathrm{pl}}\right)^{\mathrm{n}}\right]\left(1-\hat{\theta}^{\mathrm{m}}\right)
$$

Where $\bar{\varepsilon}^{\mathrm{pl}}$ is the equivalent plastic strain and $\mathrm{A}, \mathrm{B}, \mathrm{n}$ and $\mathrm{m}$ are material parameters measured at or below the transition temperature. $\hat{\theta}$ is the non-dimensional temperature expressed as a ratio between the current, transition, and melting temperatures of the material [35]. Linear tetrahedral element (C3D4) was used for meshing the foam geometry. The foam geometry had a total of 2,063,373 elements. Mie-Gruneisen Equation of State (EOS) with a linear $U_{s}-u_{p}$ was also used for both flyer-plate models. For the flyer-plates, 8-node hexahedral elements (C3D8R) were chosen for meshing. 


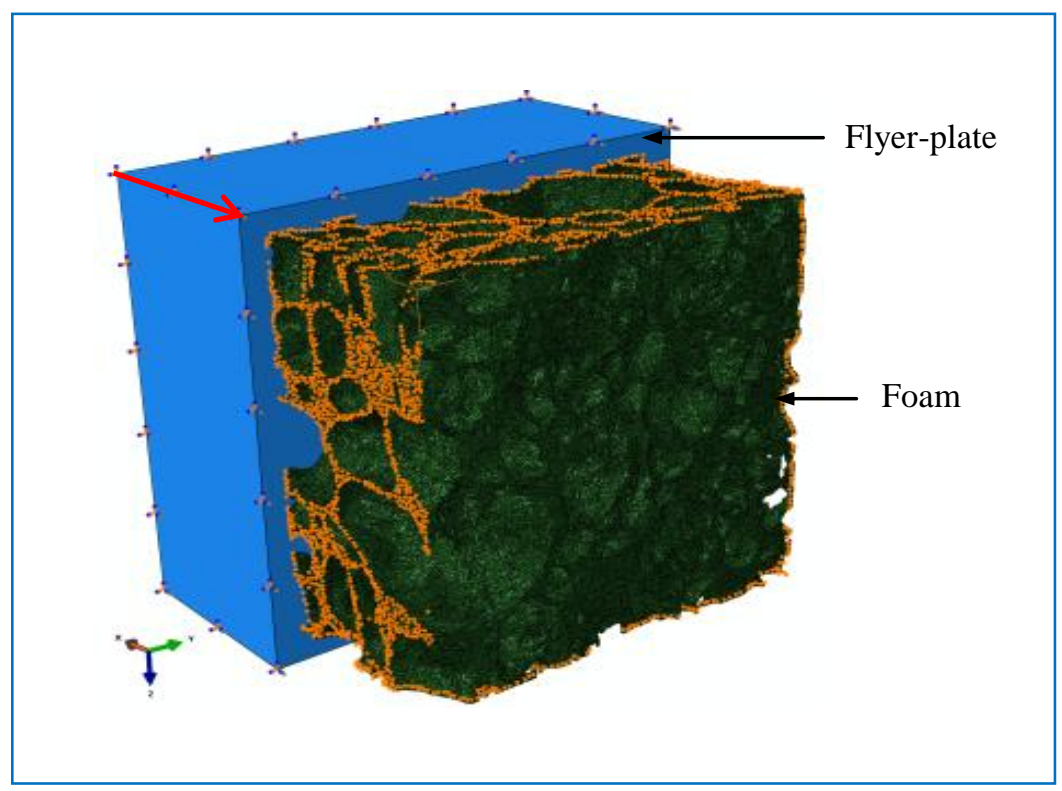

Figure 6- Meso-scale FE model of flyer-plate experiment

The impact velocity was applied to the flyer and a boundary condition was applied to the target's perimeters to mimic confinement. All degrees of freedom of the flyer-plate are restrained except in the velocity direction. The general contact option of ABAQUS/Explicit was used for interactions between the flyer and target and for all interior contacts of the foam structure. The finite element model assembly of a target foam and flyer plate is shown in Figure 6.

\section{Results and Discussions}

The experiments and FE modelling have been accomplished with three different impact velocities. To elucidate the wave propagation through the aluminium foam, a number of experiments and simulations with micro-computed tomography based geometry have been performed. Depending on intensity of velocity, the impacts were divided into two groups: medium-velocity impact $(219 \mathrm{~m} / \mathrm{s}$ and $480 \mathrm{~m} / \mathrm{s})$ and high-velocity impact $(845 \mathrm{~m} / \mathrm{s})$, for which the results will be discussed separately. 


\subsection{Flyer-plate impact with medium velocity impactors}

Plate-impact experiments and finite element simulations have been performed with two medium impact velocities of $219 \mathrm{~m} / \mathrm{s}$ and $480 \mathrm{~m} / \mathrm{s}$. In these cases, an aluminium flyer-plate was used. Figure 7 (a) shows the variation of free surface velocity with time for an impactor velocity of $219 \mathrm{~m} / \mathrm{s}$. An overall gradually increasing trend of velocity is observed from experiment as well as FE simulations. Although the slopes of the velocity curves are almost identical, a small time delay between experiment and simulation is noticed. There are two possibilities that may cause the lag. The first reason may be due to the comparatively lower computational accuracy of the tetrahedral elements that has been used to mesh the geometry. However, for more complex structures, like closed-cell aluminium foam, a little expense of accuracy may be outweighed by the advantages of meshing performance. A second possible explanation of this discrepancy is that pure aluminium as a base material was used in the modelling approach whereas the aluminium structural material contained ceramic intermetallics, trace amount of impurities and a significant portion of microporosity in the cell walls. Nevertheless, the overall FE prediction is reasonably good. It can be seen that, there is no significant velocity jump in both the experimental and numerical approaches. That is, clearly shock does not appear at $219 \mathrm{~m} / \mathrm{s}$ impact velocity. 




(a)

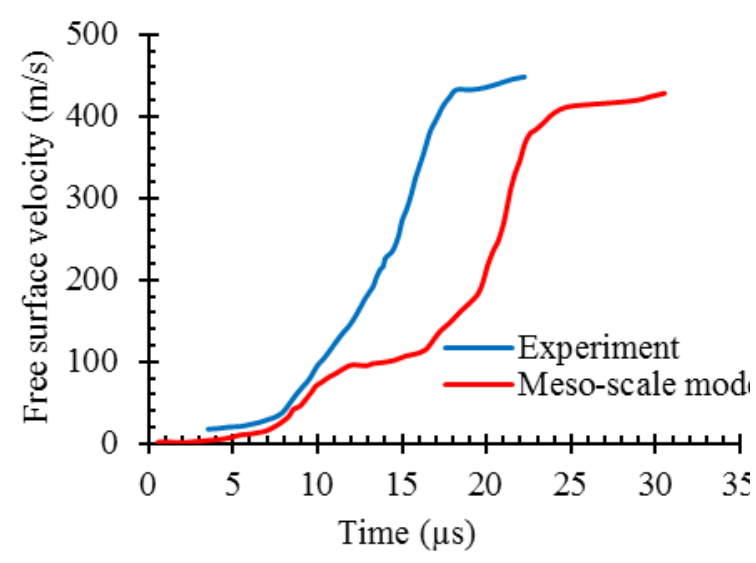

(b)

Figure 7- Comparison of free surface velocity between experiment and FE modelling for flyer-plate impact velocity (a) $210 \mathrm{~m} / \mathrm{s}$ and (b) $480 \mathrm{~m} / \mathrm{s}$ with time.

The variation of free surface velocity with time for the $480-\mathrm{m} / \mathrm{s}$ plate-impact experiment is presented in Figure 7 (b). As is shown, the predicted free surface velocity with FE modeling increases gradually up to $10 \mu$ s and agrees with the experimental result. After a short plateau at $100 \mathrm{~m} / \mathrm{s}$, the velocity again increases at a very similar rate as the experiment. However a small deviation is found in the simulation compared to the experimental results at $480 \mathrm{~m} / \mathrm{s}$ impact velocity. As already discussed the material properties used in this modelling is pure aluminum whereas the actual foam contains some impurities. These might be possible reasons of small deviation of FE prediction from experimental observations. However, except a small plateau at the beginning of plastic deformation the overall FE prediction is reasonably good with a very similar velocity gradient.

\subsubsection{Foam deformation mechanism}

The foam deformation mechanism due to impact loading has been analyzed numerically with the micro-computed tomography based digital geometry. The investigation was carried out for two different impact velocities of $219 \mathrm{~m} / \mathrm{s}$ and $480 \mathrm{~m} / \mathrm{s}$ without employing a failure model. The comparative deformation mechanism is presented with a series of sectional views in Figure 8. As is observed, for the $219 \mathrm{~m} / \mathrm{s}$ impact velocity case the cell-wall deformation 
takes place mostly at the vicinity of impactor and deforms up to a few millimeters. Particularly at the deformation region shown in the zoomed view section, the thin/weaker cell-walls deform more quickly than the thick/stronger cell-wall. Furthermore, it can be seen that the collapse occurs progressively with the source of the collapse originating from the flyer-plate / target interface. This results in a compaction wave propagating through the thickness of the material. This is unlike quasi-static compression where the collapse can occur at any point through the thickness [34].

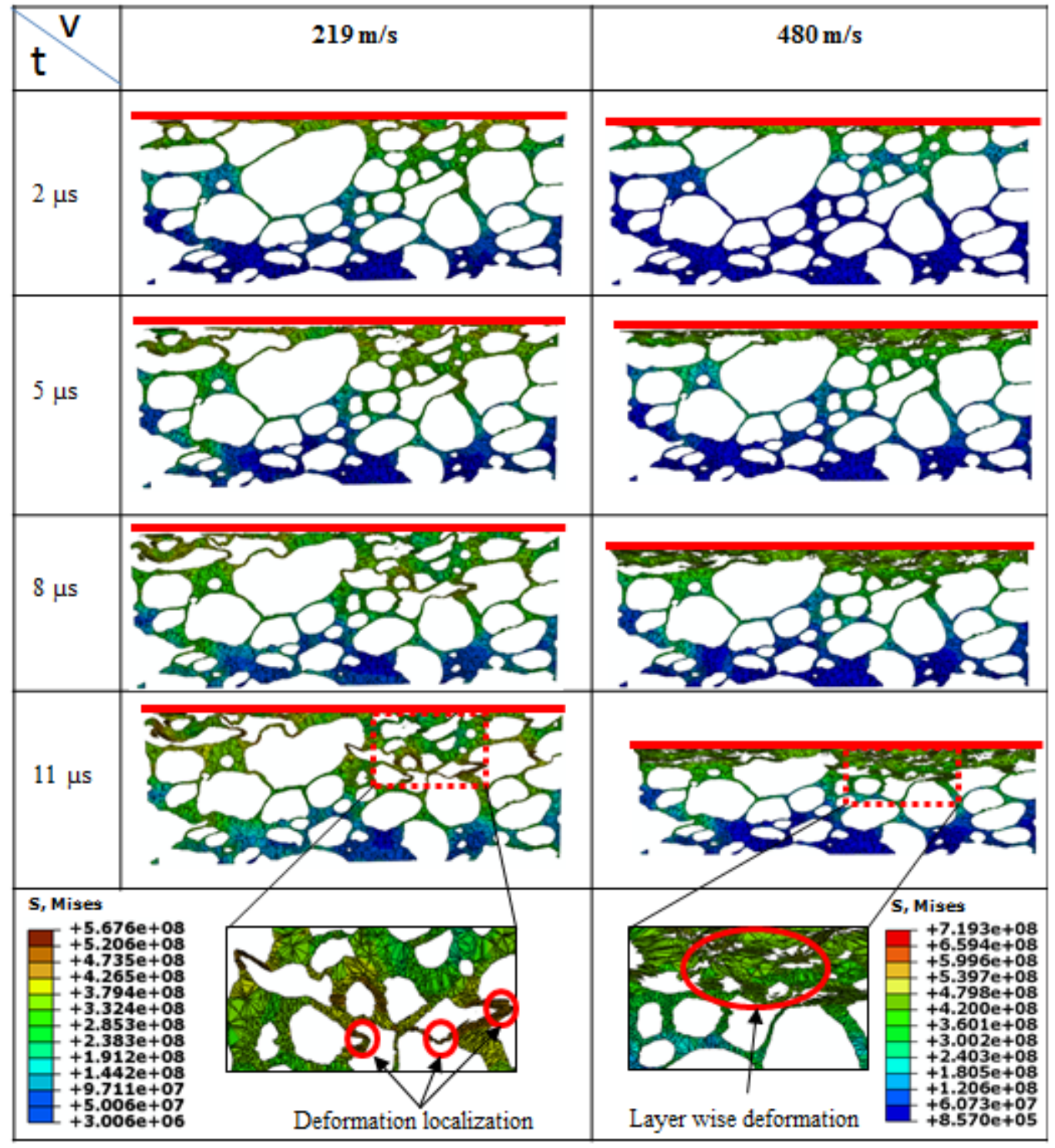

Figure 8- Plastic deformation mechanism of foam for impact velocity $219 \mathrm{~m} / \mathrm{s}$ and $480 \mathrm{~m} / \mathrm{s}$ with meso-scale modelling without damage (vertical section). 
It can be seen that, for $480 \mathrm{~m} / \mathrm{s}$ impact velocity the layer wise collapse occurs from the impact surface without systematic bending in the weaker cell walls. This is unlike the comparatively lower strain rate $(219 \mathrm{~m} / \mathrm{s})$ deformation mechanism. This prediction is consistent with the present experiment as well as previous experiment on high velocity impact on porous materials [1, 16]. Zheng et al. [31] also has shown in their voronoi cell based dynamic modelling that the foam cell deforms near to impactor. They also mentioned that, unlike quasi-static compression, more layer-wise deformation observed due to a higher impact velocity. Another research group analyzed the variation of foam deformation with their developed foam geometry (random distributed pores) and concluded that even when the deformation of the foam base materials are thought to be rate insensitive, the deformation mechanism of the foam is rate sensitive under impact loading [37].

\subsubsection{Post-impact examination}

Figure 9 (a) shows a micrograph of the experimental post-mortem sample impacted at 480 $\mathrm{m} / \mathrm{s}$ velocity. It is observed that the specimen has been compacted from $10 \mathrm{~mm}$ to $\sim 2 \mathrm{~mm}$ $(\sim 80 \%)$ in thickness during the impact.

From close observation of the micro-graph (Figure 9 b), more layer-wise compaction is noticed. The zoomed view of the fragmented foam shows that cells stack one after the other. It is considered that pore collapse during dynamic compaction was accomplished in a very short time (in few microseconds). Therefore, there was no time for systematic bending and buckling of individual struts as is observed in low strain rate compaction [13]. The micrograph of a post-mortem specimen reveals that some regions of the micro-graph are covered with cell-wall and other parts show evidence of cell collapse. Thus, it is expected that the 
deformation of closed-cell foam during plate-impact can be characterized as cell-wall fragmentation, densification and overlapping by the neighbouring wall.

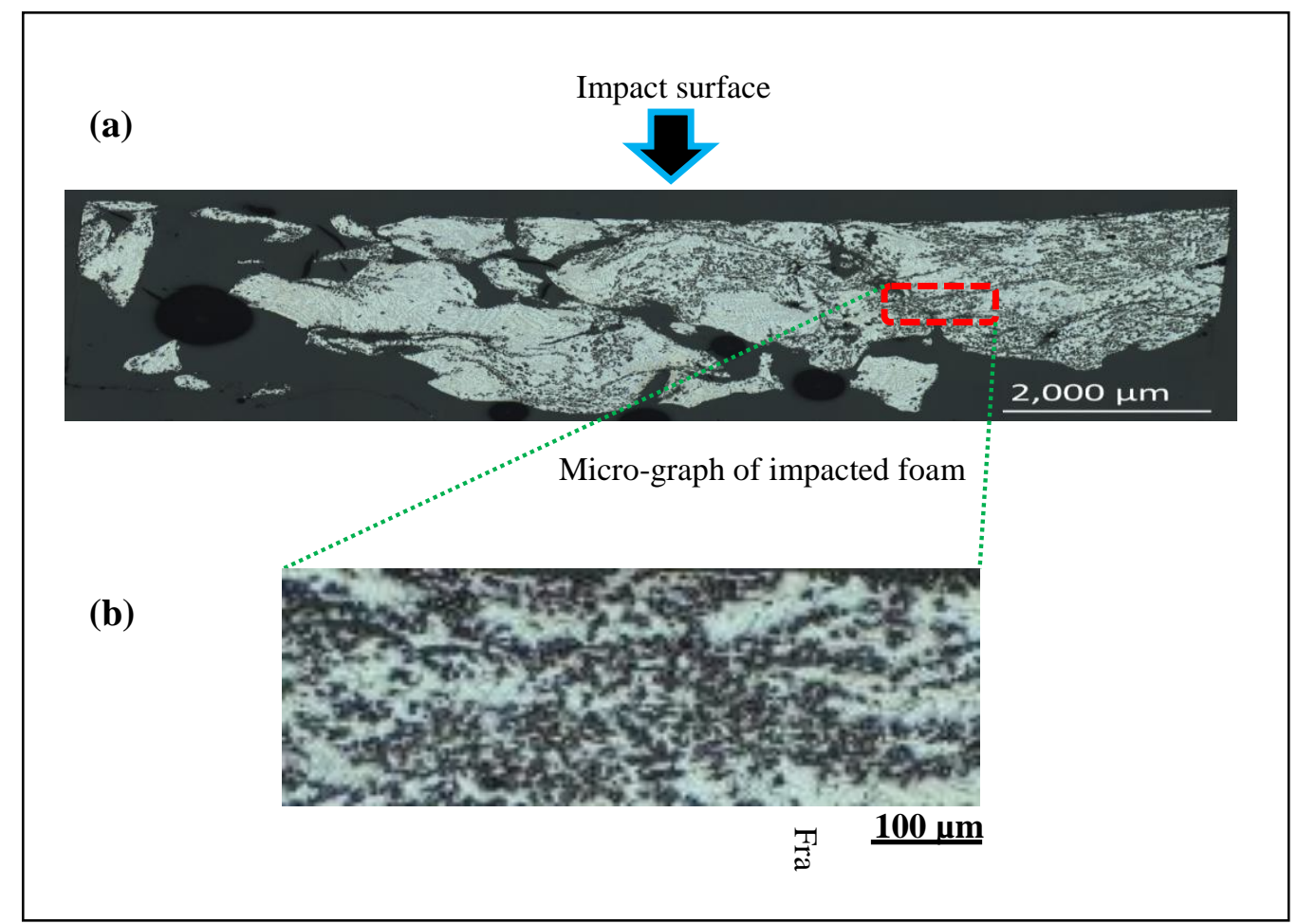

Figure 9- (a) Micrograph of post-impacted foam and (b) zoomed view of fragmented cell-walls

\subsection{Flyer-plate impact with high-velocity impactor}

Figure 10 shows the comparison of experimental recorded results and FE predictions for a flyer plate impact with a velocity of $845 \mathrm{~m} / \mathrm{s}$. As is observed, the FE predictions and experimental results show a similar ramping behaviour at the beginning of the impact process. Then a sharp rise of free surface velocity is noticed in the experiment, indicative of shock wave development. On the other hand, the FE modelling results slowly increases with a low velocity pulse at around $5 \mu$ s prior to the abrupt rise. This small jump and plateau is indicative of an elastic precursor wave. Notably this small jump appears due to the elastic wave front that propagates through convoluted pathways to reach the free surface within $5 \mu \mathrm{s}$. Petel et al. [2] also found a similar precursor wave in his plate-impact shock experiment with 
open cell aluminium foams. However, the present experimental results do not show any significant precursor-similar to the FE model. Additionally, the rear surface of the foam sample is neither perfectly planar nor flat and contains different sizes of pores with various depths. Therefore it is worth observing the variation of free surface velocity at different pores of dissimilar depth. To that end, the free surface velocity has been investigated at a number of uneven positions. As is observed, the peak velocity slightly varies with changing positions into the various sizes of pores. The peak velocity increases with increase the depth of pores. That is, increasing the pore depth reduces the foam thickness. Consequently, higher velocity is observed due to less wave attenuation.



Figure 10- Comparison of free surface velocity-time curve between experiment and FE simulations for $845 \mathrm{~m} / \mathrm{s}$ flyer plate velocity. The visual wave propagation is also correlated with velocity-time curve. 
The closest FEM free surface velocity agreement with the experiments is taken as a datum $(\mathrm{x}=0)$. Then the free surface velocities are determined at different depths of uneven surface for positive and negative $\mathrm{x}$ values ( $\mathrm{x}$ is the distances between datum plane and the plain where the velocity is measured). Positive $\mathrm{x}$ indicates the increase of thickness whereas negative $\mathrm{x}$ indicates the decrease of thickness by the mentioned values. Although the developed geometry is $8 \mathrm{~mm}$ thickness, still it is possible to get some similar planes at rear surface like the experimental sample $(10 \mathrm{~mm})$ due to the presence of pores.

The Von Mises stress is analysed with the meso-scale model to investigate the elastic-plastic deformation behaviour of the foam due to the shock wave propagation. Figure 11 shows a series of contours for the stress history in the foam target that has been stuck by a copper flyer at $845 \mathrm{~m} / \mathrm{s}$. As can be seen, when the flyer plate hits the target material, initially an elastic stress wave propagates from the top face to the bottom through convoluted pathways. This elastic wave reaches the bottom face within around $5 \mu$ s of impact. A plastic wave with a lower velocity also propagates towards the loading direction (See Figure 10). We will now discuss the wave propagation in more detail. 


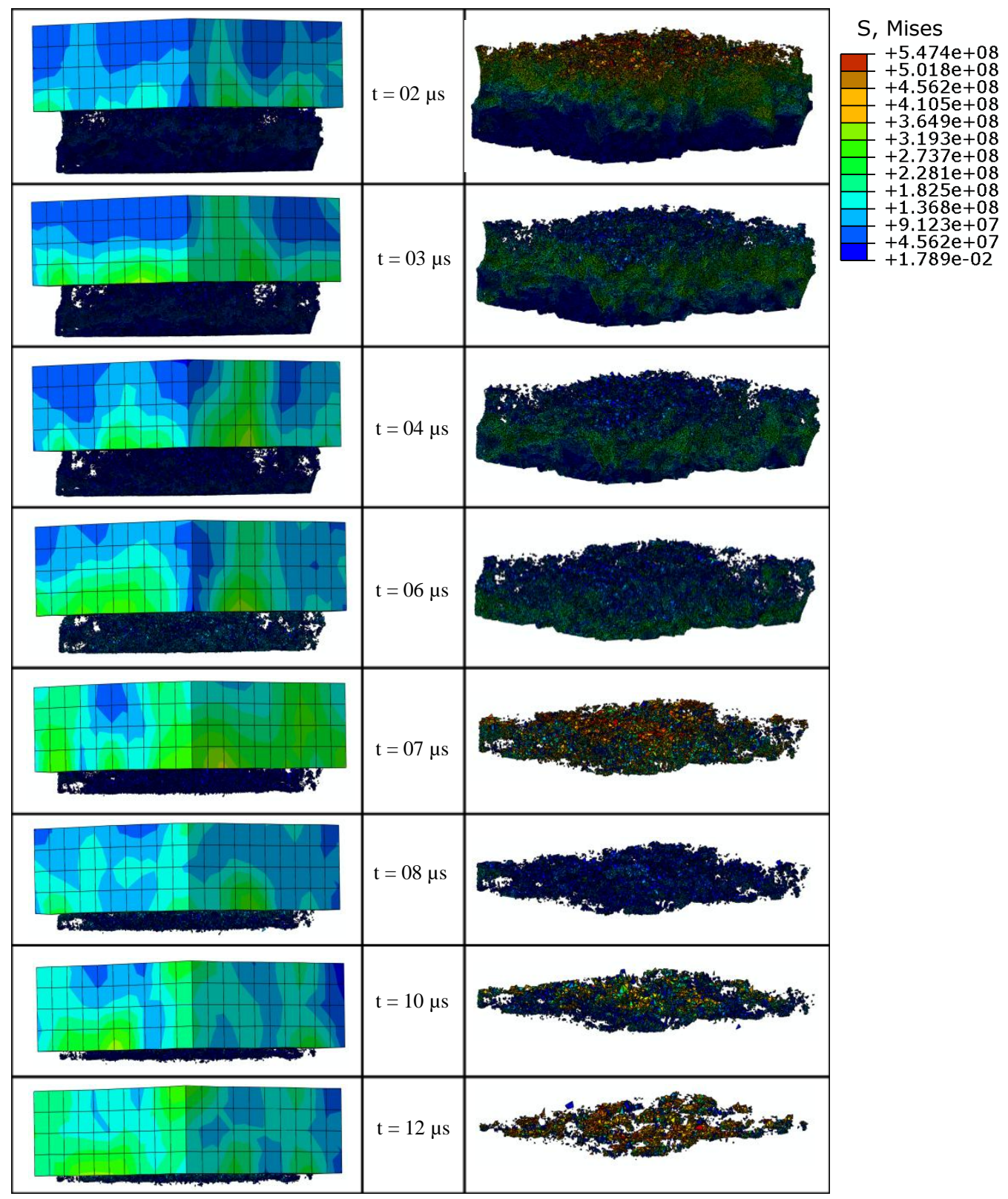

Figure 11-Deformation of foam at $845 \mathrm{~m} / \mathrm{s}$ impact velocity (Cu flyer)

\subsubsection{Wave propagation}

Figure 12 depicts the elastic and plastic wave propagation nature for shock loading throughout the loading direction. From this figure it can be seen that the elastic wave front marked with a red line is moving towards impact direction. Notably, the wave front profile is 
not straight like solid materials even though uniform loading was applied. It propagates through convoluted path depending on the nature of the foam topology. The actual distance travelled by shock wave is longer than the linear distance. The solid red line (12 mm length) shown in Figure 13 is the shortest possible path for wave propagation for this particular section whereas the dotted line shows the linear distance amounting to about $8 \mathrm{~mm}$. As observed, the wave has to propagate almost 1.5 times its linear path. From closer observation of the elastic wave front, it is found that the wave-front propagates at $\sim 1600 \mathrm{~m} / \mathrm{s}$ and reaches the rear surface at $5 \mu \mathrm{s}$. However this is far lower than the elastic wave velocity through its parent material (that amounts to $\sim 6400 \mathrm{~m} / \mathrm{s}$ ).



Figure 12-Elastic wave propagation nature due to high-velocity plate impact ( $845 \mathrm{~m} / \mathrm{s})$.

Petel et al. [2] carried out a plate impact experiment on open cell aluminium foam and reported that the elastic wave speed was $1500 \mathrm{~m} / \mathrm{s}$, which agrees well with the present simulation. Possible underlying reasons behind this are (i) actual distances travelled by the wave through the structure is larger than the linear distance, (ii) microporosity existed within the struts, reducing the stiffness of the material and (iii) the energy of the elastic wave was lost to the surrounding pores. In addition, a comparatively slower plastic wave developed and propagates following the elastic wave clearly visible in Figure 12, where the plastic wave cannot catch the elastic wave through the convoluted path. This implies that the plastic wave 
speed (shock) cannot exceed the local sonic velocity of the material and thus the wave is split into elastic and plastic wave (for this impact stress). As with condensed materials, the elastic precursor will disappear when the shock velocity is greater than or equal to the sonic velocity of a specific foam material and topology. The spatially heterogeneous nature of the shock front shown in Figure 12 indicates the importance of foam geometry to shock propagation. The foam collapse mechanism also follows the wavy nature of shock front. Notably, the arrival time of shock at the rear face of foam may vary with different positions. Therefore the designer should be careful about the foam structure and the shortest possible path for wave propagation for designing foams for ballistic and blast protection applications.



Figure 13-Vertical section of foam showing linear and actual distances travelled by wave. 


\subsubsection{Pore collapse mechanism}

The deformation and shock absorption behaviour of foam significantly relies on pore collapse pattern during impact [13]. The three dimensional pore collapse mechanisms were computationally analysed for $845 \mathrm{~m} / \mathrm{s}$ impact velocities. A single pore is considered to predict the collapse mechanics.

\section{S, Mises}

$+6.588 e+08$ $+6.039 \mathrm{e}+08$ $+5.490 \mathrm{e}+08$ $+4.941 e+08$

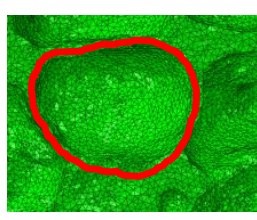

$0.00 \mu \mathrm{s}$

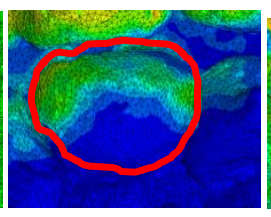

$1.08 \mu \mathrm{s}$

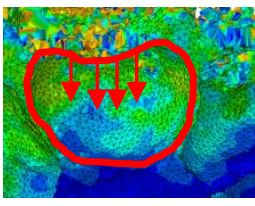

$1.80 \mu \mathrm{s}$



Leeward wall $3.00 \mu \mathrm{s}$ $+3.843 e+08$ $+3.294 e+08$ $+2.745 e+08$ $+2.196 \mathrm{e}+08$ $+2.196 \mathrm{e}+08$ $+1.647 \mathrm{e}+08$ $+1.098 \mathrm{e}+08$
$+5.490 \mathrm{e}+07$ $+5.490 \mathrm{e}+07$
$+1.559 \mathrm{e}-02$ à $3.50 \mu \mathrm{s}$

Figure 14- Pore collapse mechanism at $845 \mathrm{~m} / \mathrm{s}$ plate impact velocity with time.

The details of a pore collapse mechanism with a series of images tracking with time are shown in Figure 14. It is seen from the sequence of pore shape through time that the collapse initiates at the top surface and proceeds to consolidate the pore. It takes $3.50 \mu$ s to fully collapse and consolidate the pores at the impact interface.

As can be seen that the pore collapse in a non-uniform fashion and proceeds with the movement of the wave. The shock wave moves towards rear surface following the shortest possible way and to do so it frequently changes the direction towards nearest cell-wall shown in Figure 13. That is, the wave may deviate from its linear path and give a lateral thrust to a nearby pore. It reveals that the transmitted compressive load from neighbouring cell has a significant contribution to pore collapse. 


\section{Conclusions}

The shock propagation and elastic-plastic deformation behaviour of closed-cell aluminium foam is assessed with experimental and computational approaches. Flyer-plate impact experiments have been carried out at three different impact velocities and a micro-computed tomography geometry was developed for meso-scale modelling to track wave propagation through a realistic foam interior. Free-surface velocity histories were investigated for each impact and compared experimentally and numerically. The major findings of this work are summarized below.

(1) The free surface velocity measurements and modelling results revealed that a shock was present when the foam was struck by a copper flyer plate at an impact velocity $845 \mathrm{~m} / \mathrm{s}$ with a sudden rise of free surface velocity in the plastic deformation zone. A small amount of peak velocity variation was noticed with variation of foam thickness due to the uneven rear surface.

(2) It has been shown that the wave propagates through shortest possible path which significantly depends on foam topology. For this particular foam, the length of shortest possible path for wave propagation is 1.5 times of foam thickness. From critical observation of wave propagation it is found that the elastic wave propagates through convoluted path with $1600 \mathrm{~m} / \mathrm{s}$ velocity and reaches the rear surface within $5 \mu$ s for high velocity impact $(845 \mathrm{~m} / \mathrm{s})$. A comparatively slower velocity plastic wave propagates following the elastic wave, where the plastic wave cannot catch the elastic wave throughout the convoluted path. This observation suggests that the elastic precursor wave does not appear if the shock velocity is equal to or more than sonic velocity of a specific foam material.

(3) The FE modelling exhibits that the deformation initiates at thin/weaker cell walls with a localized mode in comparatively lower velocity regime $(219 \mathrm{~m} / \mathrm{s})$, whereas at comparatively higher velocity $(480 \mathrm{~m} / \mathrm{s})$, collapse experienced at the vicinity of the impact surface occurred 
in layers. The plastic pore collapse mechanism due to shock propagation is explored with a series of 3D digital images for $845 \mathrm{~m} / \mathrm{s}$ impact velocity. The results have indicated that an abrupt collapse initiates at the top surface and propagates to consolidate the pore into its void spaces. In addition, the transmitted compressive load with complex movements from neighbouring cell has a significant contribution to pore collapse.

\section{Acknowledgement}

The authors gratefully acknowledge the laboratory facility provided by Department of Applied Mathematics, Australian National University. We also gratefully acknowledge the UNSW Canberra Defence Related Research Program that part-funded this work.

\section{References}

[1] M. Peroni, G. Solomos, V. Pizzinato, Impact behaviour testing of aluminium foam, International Journal of Impact Engineering, 53 (2013) 74-83.

[2] O.E. Petel, S. Ouellet, D.L. Frost, A.J. Higgins, Shock Hugoniot measurements in foam. Journal of Physics: Conference Series, in, 2014.

[3] M. Saadatfar, M. Mukherjee, M. Madadi, G.E. Schröder-Turk, F. Garcia-Moreno, F.M. Schaller, S. Hutzler, A.P. Sheppard, J. Banhart, U. Ramamurty, Structure and deformation correlation of closed-cell aluminium foam subject to uniaxial compression, Acta Materialia, 60 (2012) 3604-3615.

[4] Z. Zheng, Y. Liu, J. Yu, S.R. Reid, Dynamic crushing of cellular materials: Continuumbased wave models for the transitional and shock modes, International Journal of Impact Engineering, 42 (2012) 66-79. 
[5] S.L. Lopatnikov, B.A. Gama, M.J. Haque, C. Krauthauser, J.W. Gillespie Jr, Highvelocity plate impact of metal foams, International Journal of Impact Engineering, 30 (2004) 421-445.

[6] C.Y. Zhang, L.Q. Tang, B. Yang, L. Zhang, X.Q. Huang, D.N. Fang, Meso-mechanical study of collapse and fracture behaviors of closed-cell metallic foams, Computational Materials Science, 79 (2013) 45-51.

[7] M.I. Idris, T. Vodenitcharova, M. Hoffman, Mechanical behaviour and energy absorption of closed-cell aluminium foam panels in uniaxial compression, Materials Science and Engineering: A, 517 (2009) 37-45.

[8] F.N.A. Despande V. S., Isotropic constitutive models for metallic foams, Journal of the Mechanics and Physics of Solids, 48 (2000) 1253-1283.

[9] R.P. Merrett, G.S. Langdon, M.D. Theobald, The blast and impact loading of aluminium foam, Materials \& Design, 44 (2013) 311-319.

[10] Y. Chi, G.S. Langdon, G.N. Nurick, The influence of core height and face plate thickness on the response of honeycomb sandwich panels subjected to blast loading, Materials \& Design, 31 (2010) 1887-1899.

[11] J. Banhart, Manufacture, characterisation and application of cellular metals and metal foams, Progress in Materials Science, 46 (2001) 559-632.

[12] P.J. Hazell, Armour: Materials, Theory, and Design, $1^{\text {st }}$ Edition, CRC press, Boca Raton, FL, USA, 2015.

[13] M.A. Islam, M.A. Kader, J.P. Escobedo, P.J. Hazell, G.J. Appleby-Thomas, Dynamic crushing response of closed-cell aluminium foam during shock loading, in: APS-SCCM, 2015. 
[14] P.J. Tan, S.R. Reid, J.J. Harrigan, On the dynamic mechanical properties of open-cell metal foams - A re-assessment of the 'simple-shock theory', International Journal of Solids and Structures, 49 (2012) 2744-2753.

[15] M.A. Kader, M.A. Islam, P.J. Hazell, J.P. Escobedo, M. Saadatfar, A.D. Brown, Numerical modelling of closed-cell aluminium foams under shock loading in: APS-SCCM, 2015.

[16] P.J. Tan, S.R. Reid, J.J. Harrigan, Z. Zou, S. Li, Dynamic compressive strength properties of aluminium foams. Part I-experimental data and observations, Journal of the Mechanics and Physics of Solids, 53 (2005) 2174-2205.

[17] S.L. Lopatnikov, B.A. Gama, M. Jahirul Haque, C. Krauthauser, J.W. Gillespie Jr, M. Guden, I.W. Hall, Dynamics of metal foam deformation during Taylor cylinder-Hopkinson bar impact experiment, Composite Structures, 61 (2003) 61-71.

[18] M. Vasenjak, M. Borovinsek, Z. Ren, S. Irie, S. Itoh, Behaviour of metallic foam under shock wave loading, Metals, 2 (2012) 258-264.

[19] D.D. Radford, V.S. Deshpande, N.A. Fleck, The use of metal foam projectiles to simulate shock loading on a structure, International Journal of Impact Engineering, 31 (2005) $1152-1171$

[20] M.W. Seitz, B.W. Skews, An analytical model for shock wave impact on compressible open-cell foam, Shock Waves, 16 (2007) 287-298.

[21] P.J. Tan, Harrigan J. J., S.R. Reid, Inertia effects in the uniaxial dynamic compression of a closed-cell aluminium alloy foam, Material Science and Technology, 18 (2002) 480-488.

[22] V.R. Feldgun, Y.S. Karinski, D.Z. Yankelevsky, A two-phase model to simulate the 1-D shock wave propagation in porous metal foam, International Journal of Impact Engineering, $82(2015)$ 113-129. 
[23] P.J. Hazell, G.J. Appleby-Thomas, E. Wielewski, C. Stennett, C. Siviour, The influence of microstructure on the shock and spall behaviour of the magnesium alloy, Elektron 675, Acta Materialia, 60 (2012) 6042-6050.

[24] S.J. Fensin, J.P. Escobedo-Diaz, C. Brandl, E.K. Cerreta, G.T. Gray Iii, T.C. Germann, S.M. Valone, Effect of loading direction on grain boundary failure under shock loading, Acta Materialia, 64 (2014) 113-122.

[25] L. Bodelot, J.P. Escobedo-Diaz, C.P. Trujillo, D.T. Martinez, E.K. Cerreta, G.T. Gray, G. Ravichandran, Microstructural changes and in-situ observation of localization in OFHC copper under dynamic loading, International Journal of Plasticity, 74 (2015) 58-74.

[26] J.P. Escobedo, E.N. Brown, C.P. Trujillo, E.K. Cerreta, G.T. Gray III, The effect of shock-wave profile on dynamic brittle failure, Journal of Applied Physics, 113 (2013) 103506.

[27] J.P. Escobedo, E.K. Cerreta, D. Dennis-Koller, C.P. Trujillo, C.A. Bronkhorst, Influence of boundary structure and near neighbor crystallographic orientation on the dynamic damage evolution during shock loading, Philosophical Magazine, 93 (2013) 833-846.

[28] P. Hazell, G. Appleby-Thomas, E. Wielewski, J. Escobedo, The shock and spall response of three industrially important hexagonal close-packed metals: magnesium, titanium and zirconium, Philosophical Transactions of the Royal Society of London A: Mathematical, Physical and Engineering Sciences, 372 (2014) 20130204.

[29] M. Goff, P.J. Hazell, G.J. Appleby-Thomas, D.C. Wood, C. Stennett, P. Taylor, Gas gun ramp loading of Kel-F 81 targets using a ceramic graded areal density flyer system, International Journal of Impact Engineering, 80 (2015) 152-161.

[30] Y. Zhang, G. Sun, X. Xu, G. Li, X. Huang, J. Shen, Q. Li, Identification of material parameters for aluminum foam at high strain rate, Computational Materials Science, 74 (2013) 65-74. 
[31] Z. Zheng, C. Wang, J. Yu, S.R. Reid, J.J. Harrigan, Dynamic stress-strain states for metal foams using a 3D cellular model, Journal of the Mechanics and Physics of Solids, 72 (2014) 93-114.

[32] N.K. Bourne, K. Bennett, A.M. Milne, S.A. MacDonald, J.J. Harrigan, J.C.F. Millett, The shock response of aluminium foams, Scripta Materialia, 58 (2008) 154-157.

[33] M.A. Islam, J.P. Escobedo, P.J. Hazell, G.J. Appleby-Thomas, M.Z. Quadir, Characterization of Closed-Cell Aluminium Foams Subjected to Compressive Loading, in: TMS Annual Meeting, 2015, pp. 167-174.

[34] M. Islam, P. Hazell, J. Escobedo, M. Saadatfar, In-Situ Quasistatic Compression and Microstructural Characterization of Aluminium Foams of Different Cell Topology, in: XII International Conference on Applied Mechanics and Mechanical Engineering (ICAMME 2014:), 2014, pp. 11-12.

[35] ABAQUS Technology Brief, Simulation of the ballistic perforation of aluminium plates with Abaqus/Explicit, Dassault Systemes, in, 2012.

[36] G.R. Johnson, W. Cook, Fracture characteristics of three metals subjected to various strains, strain rates, temperatures and pressures, Engineering Fracture Mechanics, 21 (1985) $31-48$.

[37] Q. Fang, J. Zhang, Y. Zhang, H. Wu, Z. Gong, A 3D mesoscopic model for the closedcell metallic foams subjected to static and dynamic loadings, International Journal of Impact Engineering, 82 (2015) 103-112. 